\title{
ON THE NUMERICAL SOLUTION OF PERTURBED BIFURCATION PROBLEMS
}

\section{B. M. ELGINDI and R. W. LANGER}

Department of Mathematics University of Wisconsin - Eau Claire Eau Claire, WI 54702

(Received February 3, 1993 and in revised form March 22, 1994)

\begin{abstract}
Some numerical schemes, based upon Newton's and chord methods, for the computations of the perturbed bifurcation points as well as the solution curves through them, are presented. The "initial" guesses for Newton's and chord methods are obtained using the local analysis techniques and proved to fall into the neighborhoods of contraction for these methods. In applications the "perturbation" parameter represents a physical quantity and it is desirable to use it to parameterize the solution curves near the perturbed bifurcation point. In this regard, it is shown that, for certain classes of the perturbed bifurcation problems, Newton's and chord methods can be used to follow the solution curves in a neighborhood of the perturbed bifurcation point while the perturbation parameter is kept fixed.
\end{abstract}

KEY WORDS: Perturbed bifurcation, singular perturbation, bifurcation from the trivial solution, Newton's and chord methods.

1980 AMS SUBJECT CLASSIFICATION CODES: 65J99, 58F14.

\section{INTRODUCTION}

Many problems in applications are formulated as

$$
G(x, \lambda, \tau)=0
$$

where $G: H \times \mathbf{R} \times \mathbf{R} \rightarrow H$ is a smooth mapping, $H$ is a real Hilbert space, $\lambda$ and $\tau$ are real parameters often representing some physical quantities, $G$ satisfies the properties

$$
\begin{array}{ll}
G(0, \lambda, 0)=0, & \lambda \in \mathbf{R}, \\
G_{\tau}(0, \lambda, 0) \neq 0, & \lambda \in \mathbf{R},
\end{array}
$$

and the Frechet derivative $G_{x}^{0} \equiv G_{x}\left(0, \lambda_{0}, 0\right)$ satisfies for some $\lambda_{0} \in \mathbf{R}$

(a) $N\left(G_{x}^{0}\right)$ is one-dimensional spanned by $\phi,\langle\phi, \phi\rangle=1$,

(b) $N\left(G_{x}^{0^{*}}\right)$ is one-dimensional spanned by $\psi \neq 0$,

(c) $R\left(G_{x}^{0}\right)=N\left(G_{x}^{0^{*}}\right)^{1}$ and $R\left(G_{x}^{0^{*}}\right)=N\left(G_{x}^{0}\right)^{1}$,

(d) $a \equiv\left\langle\psi, G_{x \lambda}^{0} \phi\right\rangle \neq 0$,

(e) $\langle\psi, \phi\rangle=1$,

(f) $b \equiv\left\langle\psi, G_{\tau}^{0}\right\rangle \neq 0$,

where $G_{x}^{0^{*}}$ denotes the adjoint operator of $G_{x}^{0}, G_{x \lambda}^{0} \equiv G_{x \lambda}\left(0, \lambda_{0}, 0\right)$ and $G_{\tau}^{0} \equiv G_{\tau}\left(0, \lambda_{0}, 0\right)$.

Properties (1.2) and (1.3) respectively imply that the "trivial" solution $x=0$ solves the "unperturbed" problem

$$
G(x, \lambda, 0)=0,
$$

for each $\lambda \in \mathbf{R}$ and that it is not a solution of (1.1) for any $\lambda \in \mathbf{R}$ when $\tau \neq 0$. Properties (a)-(c) of (1.4) imply that $G_{x}^{0}$ is a Fredholm operator with zero index and together with the property (d) of (1.4) imply 
that $\left(0, \lambda_{0}, 0\right)$ is a bifurcation point of (1.5). Observe also that property (e) of (1.4) implies that the zero eigenvalue of $G_{1}^{0}$ has algebraic multuplicity one.

The parameters $\lambda$ and $\tau$ in (1.1) are usually called the bifurcation parameter and the perturbation parameter respectively. When $\tau \neq 0$, the solution set of (1.1) is completely different from that of (1.5) and in this case (1.1) is usually termed a perturbed bifurcation problem. Many excellent analytical and numerical treatments of such problems exist in the literature. The reader is referred to [4], [6], [7], [9], [10], [11] and the references therein for an extensive account of the subject.

In this paper we concentrate on the numerical aspect of (1.1) and in particular on the applications of Newton's and chord methods to this problem. In this respect our techniques go along the same lines as those of Decker and Keller [3] for the bifurcation problem (1.5). The difficulties arising in the applications of Newton's and chord methods to $(1.1)$ in the neighborhood of $\left(0, \lambda_{0}, 0\right)$ are due to the nonuniqueness of the solution, the singularity (or the near singularity) of $G_{\mathrm{r}}$ and the non-availability of the "close enough" initial guesses for these methods.

The rest of this paper is organized in four sections. Some preliminary results regarding the solution set of (1.1) and based upon the Implicit Function Theorem are presented in Section 2. Also in Section 2 we state the basic convergence theorem for Newton's and chord methods which is used in the later sections. In Section 3 we present and prove the convergence of some numerical schemes for computing the perturbed bifurcation points and the solution curves through them. In Section 4 we show that Newton's and chord methods can be used to compute all solution curves of $(1.1)$ near $\left(0, \lambda_{0}, 0\right)$ for certain types of problems, while the perturbation parameter $\tau$ is kept fixed. In Section 5 we apply the schemes developed in Sections 3 and 4 to a numerical example.

\section{PRELIMINARIES}

In this section we study the behavior of the bifurcation point $\left(0, \lambda_{0}, 0\right)$ of $(1.5)$ under the perturbation $\tau \neq 0$. It is shown that there is locally a family of "limit points" through each of which there is a family of solutions of (1.1). The proof of these statements is based upon the Implicit Function Theorem. We also state the basic convergence result for Newton's and chord methods which will be used in the later sections.

Let $Y$ denote the Hilbert space $H \times H \times \mathbf{R} \times \mathbf{R}$ and $F: Y \times \mathbf{R} \rightarrow Y$ be the mapping

$$
F(y, \varepsilon)=\left[\begin{array}{l}
G\left(\varepsilon \phi+y_{1}, \lambda_{0}+r_{1}, r_{2}\right) \\
G_{x}\left(\varepsilon \phi+y_{1}, \lambda_{0}+r_{1}, r_{2}\right)\left(\phi+y_{2}\right) \\
\left\langle\phi, y_{1}\right\rangle \\
\left\langle\phi, y_{2}\right\rangle
\end{array}\right],
$$

where $y=\left(y_{1}, y_{2}, r_{1}, r_{2}\right) \in Y$, and $\varepsilon$ is a real parameter. We consider the equation

$$
F(y, \varepsilon)=0
$$

We observe that $(0,0)$ is a solution of $(2.2)$ and that the Frechet derivative of $F$ with respect to $y$ at $(0,0)$ is

$$
F_{v}(0,0)=\left[\begin{array}{cccc}
G_{x}^{0} & 0 & 0 & G_{\tau}^{0} \\
G_{x x}^{0} \phi & G_{x}^{0} & G_{x \lambda}^{0} \phi & G_{x \tau}^{0} \phi \\
\langle\phi, \cdot\rangle & 0 & 0 & 0 \\
0 & \langle\phi, \cdot\rangle & 0 & 0
\end{array}\right],
$$

where $G_{x x}^{0}, G_{x \tau}^{0}$ are $G_{x x}\left(0, \lambda_{0}, 0\right)$ are $G_{x \tau}\left(0, \lambda_{0}, 0\right)$ respectively.

LEMMA 2.1. There exists a unique smooth curve $y=y(\varepsilon)$ defined on $|\varepsilon| \leq \varepsilon_{0}$, for some $\varepsilon_{0}>0$, such that $y(0)=0$ and $F(y(\varepsilon), \varepsilon)=0$ for each $\varepsilon$ with $|\varepsilon| \leq \varepsilon_{0}$. 
PROOF. We observe that if $y=\left(y_{1}, y_{2}, r_{1}, r_{2}\right) \in Y$ satisfies

$$
F_{1}(0,0) y=0 \text {, }
$$

where $F_{1}(0,0)$ is defined by $(2.3)$, then the components of $y$ satisfy the system of equations

(a) $G_{\lambda}^{0} y_{1}+r_{2} G_{\tau}^{0}=0$

(b) $G_{\mathrm{u}}^{0} y_{1}+G_{\mathrm{r}}^{0} y_{2}+r_{1} G_{\mathrm{\imath} \lambda}^{0} \phi+r_{2} G_{\mathrm{r \tau}}^{0} \phi=0$,

(c) $\left\langle\phi, y_{1}\right\rangle=0$,

(d) $\left\langle\phi, y_{2}\right\rangle=0$.

From (a) it follows that $r_{2} b=0$, and therefore condition (f) of (1.4) implies that $r_{2}=0$, and hence $y_{1}=A \phi$, for some constant $A$. Now by (c), $A$ must be zero and hence $y_{1}=0$. This reduces (b) to $G_{\lambda}^{0} y_{2}+r_{1} G_{x \lambda}^{0} \phi=0$, which in turn implies that $r_{1} a=0$. Since $a \neq 0$ by condition (d) of (1.4), it follows that $r_{1}=0$, and that $y_{2}=A \phi$, for some constant $A$. This together with (d) implies that $y_{2}=0$. Thus, $y=0$. It follows that $F_{v}(0,0)$ is one to one. Since $F_{v}(0,0)$ is clearly onto, it follows from the Open Mapping Theorem that it has a bounded inverse. This enables us to apply the Implicit Function Theorem to equation (2.2) and obtain the desired conclusions.

Let $\varepsilon_{0}>0$ be the number provided by Lemma 2.1. Then for each $\varepsilon$ in $|\varepsilon| \leq \varepsilon_{0}$ there exist $y_{1}(\varepsilon), y_{2}(\varepsilon) \in H$ and $r_{1}(\varepsilon) \in \mathbf{R}$ such that $x(\varepsilon)=\varepsilon \phi+y_{1}(\varepsilon), \phi(\varepsilon)=\phi+y_{2}(\varepsilon), \lambda(\varepsilon)=\lambda_{0}+r_{1}(\varepsilon)$ and $\tau(\varepsilon)=r_{2}(\varepsilon)$ satisfy

$$
\begin{aligned}
G(x(\varepsilon), \lambda(\varepsilon), \tau(\varepsilon)) & =0, \\
G_{x}(x(\varepsilon), \lambda(\varepsilon), \tau(\varepsilon)) \phi(\varepsilon) & =0 .
\end{aligned}
$$

It follows from the perturbation theory for linear operators [5] that for small enough $\varepsilon_{0}$

(i) $\quad R\left(G_{x}(x(\varepsilon), \lambda(\varepsilon), \tau(\varepsilon))\right.$ is closed.

(ii) $\quad N\left(G_{x}(x(\varepsilon), \lambda(\varepsilon), \tau(\varepsilon))\right)$ is one-dimensional spanned by $\phi(\varepsilon)$,

(iii) $\quad N\left(G_{x}^{*}(x(\varepsilon), \lambda(\varepsilon), \tau(\varepsilon))\right)$ is one-dimensional, say, spanned by $\psi(\varepsilon)$,

(iv) $\quad a(\varepsilon) \equiv\left\langle\psi(\varepsilon), G_{x \lambda}(x(\varepsilon), \lambda(\varepsilon), \tau(\varepsilon)) \phi(\varepsilon)\right\rangle \neq 0$.

Now for each $\varepsilon$ in $|\varepsilon| \leq \varepsilon_{0}$, (i)-(iv) of (2.5) imply that there exists a unique smooth solution branch $\Gamma(\varepsilon)$ of (1.1) which passes through $(x(\varepsilon), \lambda(\varepsilon), \tau(\varepsilon))$. This shows that a bifurcation occurs at $(x(\varepsilon), \lambda(\varepsilon), \tau(\varepsilon))$. It follows from the perturbation theory of [7] that

$$
d(\varepsilon) \equiv\left\langle\psi(\varepsilon), G_{\lambda}(x(\varepsilon), \lambda(\varepsilon), \tau(\varepsilon))\right\rangle=a \varepsilon+0\left(\varepsilon^{2}\right),
$$

which in turn implies that this "perturbed" bifurcation point is a limit point for small enough $\varepsilon \neq 0$.

We summarize the conclusions of the above paragraph in the following theorem.

THEOREM 2.2. There exists a unique smooth curve $(x(\varepsilon), \lambda(\varepsilon), \tau(\varepsilon))$ defined on $|\varepsilon| \leq \varepsilon_{0}$, for some $\varepsilon_{0}>0$, of solutions of (1.1) passing through the bifurcation point $\left(0, \lambda_{0}, 0\right)$. Furthermore, for $\varepsilon \neq 0,(x(\varepsilon), \lambda(\varepsilon), \tau(\varepsilon))$ is a limit point through which there passes a unique smooth solution branch $\Gamma(\varepsilon)$ of (1.1).

REMARK 2.3. The use of the Implicit Function Theorem in the treatment of the perturbed bifurcation problems of the type considered in this paper was suggested in [7] where a different technique was used. 
The following basic result for the convergence of Newton's and chord methods is a consequence of Newton-Kantorovich Theorem and its statement is due to Moore [10].

THEOREM 2.4. Let $F(U, \delta)$ be a mapping from $B_{1} \times \mathbf{R}$ to $B_{2}$, where $B_{1}$ and $B_{2}$ are Banach spaces, which is continuously differentiable in $U$ and continuous in $\delta$. Let $U^{0}(\delta)$ be a continuous mapping from $\left(0, \delta_{1}\right)$ to $B_{1}$ for some $\delta_{1}>0$.

(I) Assume that for $\delta \in\left(0, \delta_{1}\right), F_{U}\left(U^{0}(\delta), \delta\right)$ has a bounded inverse which satisfies

(a) $\left\|F_{U}^{-1}\left(U^{0}(\delta), \delta\right) F\left(U^{0}(\delta), \delta\right)\right\| \leq \eta(\delta)$,

(b) $\left\|F_{U}^{-1}\left(U^{0}(\delta), \delta\right)\left[F_{U}(U, \delta)-F_{U}(V, \delta)\right]\right\| \leq L(\delta)\|U-V\|$,

for $U, V \in B_{2 \eta(\delta)}(U(\delta))$,

(c) $h(\delta) \equiv \eta(\delta) L(\delta)=0(\delta)$ as $\delta \rightarrow 0$.

Then there exists $\delta_{2}>0, \delta_{2} \leq \delta_{1}$ and a continuous mapping $U^{*}(\delta)$ from $\left(0, \delta_{2}\right)$ into $B_{1}$ such that $U^{*}(\delta)$ is the unique solution of $F(U, \delta)=0$ in the ball $B_{2 \eta(\delta)}\left(U^{0}(\delta)\right)$ and Newton's iterates.

$$
\begin{aligned}
U^{(k+1)} & =U^{(k)}-F_{U}^{-1}\left(U^{(k)}, \delta\right) F\left(U^{(k)}, \delta\right), \quad k \geq 0, \\
U^{(0)} & =U^{0}(\delta),
\end{aligned}
$$

converge to $U^{*}(\delta)$ for each $\delta \in\left(0, \delta_{2}\right)$.

(II) Assume that for $\delta \in\left(0, \delta_{1}\right), F_{U}\left(U^{0}(\delta), \delta\right)$ has a bounded inverse which satisfies condition (a) above and the conditions,

(d) $\left\|F_{U}^{-1}\left(U^{0}(\delta), \delta\right)\left[F_{U}\left(U^{0}(\delta), \delta\right)(U-V)-(F(U, \delta)-F(V, \delta))\right]\right\| \leq q(\delta)\|U-V\|$, for $U, V \in B_{2 \eta(\delta)}\left(U^{0}(\delta)\right)$,

(e) $q(\delta)=0(\delta)$ as $\delta \rightarrow 0$.

Then there exists $\delta_{2}>0, \delta_{2} \leq \delta_{1}$ and a continuous mapping $U^{*}(\delta)$ from $\left(0, \delta_{2}\right)$ into $B_{1}$ such that $U^{*}(\delta)$ is the unique solution of $F(U, \delta)=0$ in the ball $B_{2 \eta(\delta)}\left(U^{0}(\delta)\right)$ and the chord iterates

$$
\begin{aligned}
U^{(k+1)} & =U^{(k)}-F_{U}^{-1}\left(U^{(0)}, \delta\right) F\left(U^{(k)}, \delta\right), \\
U^{(0)} & =U^{0}(\delta),
\end{aligned}
$$

converges to $U^{*}(\delta)$ for each $\delta \in\left(0, \delta_{2}\right)$.

\section{THE NUMERICAL COMPUTATION OF THE PERTURBED BIFURCA- TION POINTS AND THE SOLUTION BRANCHES THROUGH THEM}

In this section we demonstrate the application of Newton's and chord methods in the computations of the perturbed bifurcation points of (1.1) and the solution branches through them.

The computations of the perturbed bifurcation points of (1.1) using the Newton's and chord iterates involve the application of Theorem 2.4 to the equations (2.2). As an "initial" guess we take $y^{(0)}=0$. It follows from the proof of Lemma 2.1 that $F_{、}(0, \varepsilon)$ has a bounded inverse, for $\varepsilon$ in $|\varepsilon| \leq \varepsilon_{0}$, where $\varepsilon_{0}>0$ is small enough. Using similar arguments as those in the proof of Lemma 2.1, we can show that $\eta(\varepsilon)$, $L(\varepsilon)$ and $g(\varepsilon)$ of Theorem 2.4 are $0(\varepsilon), 0(1)$ and $0(\varepsilon)$ as $\varepsilon \rightarrow 0$, respectively. This proves the following theorem.

THEOREM 3.1. There exists $\varepsilon_{0}>0$ such that for $0<|\varepsilon| \leq \varepsilon_{0}$ both Newton's and chord iterates with initial guess $y^{(0)}=0$ converge to the unique solution $y(\varepsilon)$ of equation (2.2).

We now consider the application of Theorem 2.4 in computing the solution branches through the perturbed bifurcation points. To this end let us assume that the unique solution $y(\varepsilon) \equiv\left(Y_{1}^{*}, y_{2}^{*}, r_{1}^{*}, r_{2}^{*}\right)$ of (2.2) corresponding to some $\varepsilon$ in $0<|\varepsilon| \leq \varepsilon_{0}$ has been determined. With $\tau=r_{2}^{*}$ being fixed we want to 
compute $(x, \lambda)$ near $\left(x^{*}, \lambda^{*}\right)$, where $x^{*}=\varepsilon \phi+y_{1}^{*}$ and $\lambda^{*}=\lambda_{0}+r_{1}^{*}$, such that

$$
G(x, \lambda, \tau)=0 \text {. }
$$

We define for $U=\left(\begin{array}{l}w \\ \mu\end{array}\right) \in H \times \mathbf{R}$ and $\delta \in \mathbf{R}$

$$
K(U, \delta)=\left[\begin{array}{l}
G\left(x^{*}+\delta \phi^{*}+w, \lambda^{*}+\mu, \tau\right) \\
\left\langle\Phi^{*}, w\right\rangle
\end{array}\right]
$$

where $\phi^{*}=\phi+y_{2}^{*}$, and consider the equation

$$
K(U, \delta)=0
$$

Since

$$
K_{U}(0,0)=\left[\begin{array}{cc}
G_{x}\left(x^{*}, \lambda^{*}, \tau\right) & G_{\lambda}\left(x^{*}, \lambda^{*}, \tau\right) \\
\left\langle\phi^{*}, \cdot\right\rangle & 0
\end{array}\right],
$$

it follows from (2.6) that $K_{U}(0,0)$ has a bounded inverse and hence the same is true for $K_{U}(0, \delta)$ for all $\delta$ in $|\delta| \leq \delta_{0}$, provided that $\delta_{0}$ is small enough. Also we have

$$
K(0, \delta)=0(\delta) \text {. }
$$

Using this, similar arguments to those used in the proof of Lemma 2.1 verify conditions (a)-(e) of Theorem 2.4 with initial guess $U^{(0)}=0$. This completes the proof of the following theorem.

THEOREM 3.2. There exists $\delta_{0}>0$ such that for each $\delta$ in $0<|\delta| \leq \delta_{0}$ Newton's and chord iterates converge to a unique solution $U^{*}(\delta)$ of (3.3).

REMARK 3.3. In some cases (1.1) may have solution curves which do not pass through the singular solutions $(x(\varepsilon), \lambda(\varepsilon), \tau(\varepsilon))$. Such solution curves cannot be determined by the above method. Under some additional assumptions it is shown in the next section that Newton's and chord methods can be used to compute all the solution curves in some neighborhood of $\left(0, \lambda_{0}, 0\right)$. The initial guesses in these cases are obtained using the singular perturbation methods [8].

\section{THE NUMERICAL COMPUTATIONS OF THE SOLUTION CURVES OF SOME PERTURBED BIFURCATION PROBLEMS}

This section is concerned with the applications of Newton's and chord methods in computing all the solution curves of $(1.1)$ in some neighborhood of $\left(0, \lambda_{0}, 0\right)$ for a given small value of the perturbation parameter $\tau \neq 0$. We will consider the following two cases.

Case 1: (The non-degenerate case) $E_{0} \neq 0$, where $E_{0} \equiv \frac{1}{2}\left\langle\psi, G_{x x}^{0} \phi \phi\right\rangle$

Case 2: (The degenerate case) $E_{0}=0, E_{1} \neq 0$ and $\frac{a}{E_{1}}<0$, where

$$
\begin{gathered}
E_{1}=\frac{2}{3}\left\langle\psi, G_{x x}^{0} \phi w_{1}\right\rangle+\frac{1}{3}\left\langle\psi, G_{x x}^{0} w_{1} \phi\right\rangle+\frac{1}{6}\left\langle\psi, G_{x x x}^{0} \phi \phi \phi\right\rangle, \\
G_{x x x}^{0}=G_{x x x}\left(0, \lambda_{0}, 0\right) \text { and } w_{1}=-G_{x}^{0-1}\left(G_{x x}^{0} \phi \phi-\left\langle\psi, G_{x x}^{0} \phi \phi\right\rangle \frac{\psi}{\|\psi\|^{2}}\right) .
\end{gathered}
$$

The singular perturbation theory of [8] reveals the following information about the solution curves of (1.1) near the bifurcation point $\left(0, \lambda_{0}, 0\right)$ for small $\tau \neq 0$. The solution curves can be written as

Case 1: $\quad x(\tilde{\varepsilon})=A \tilde{\varepsilon} \phi+0\left(\tilde{\varepsilon}^{2}\right)$,

$$
\begin{aligned}
\lambda(\tilde{\varepsilon}) & =\lambda_{0}+\xi \tilde{\varepsilon}+0\left(\tilde{\varepsilon}^{2}\right), \\
\tilde{\varepsilon}^{2} & =|\tau|,
\end{aligned}
$$


and $A$ is determined by the equation

$$
E_{0} A^{2}+a \xi A+b=0
$$

Case 2: $\quad x(\tilde{\varepsilon})=A \tilde{\varepsilon} \phi+A^{2} \tilde{\varepsilon}^{2} w_{1}+0\left(\tilde{\varepsilon}^{3}\right)$,

$$
\begin{aligned}
\lambda(\tilde{\varepsilon}) & =\lambda_{0}+\xi \tilde{\varepsilon}^{2}+0\left(\tilde{\varepsilon}^{3}\right), \\
\tilde{\varepsilon}^{3} & =|\tau|,
\end{aligned}
$$

and $A$ is determined by the equation

$$
E_{1} A^{3}+a \xi A+b=0 .
$$

Let $H_{1}$ denote the Hilbert space $H \times \mathbf{R}$ and define

$$
\begin{gathered}
F^{(t)}: H_{1} \times \mathbf{R} \times \mathbf{R} \rightarrow H_{1}, \quad i=1,2, \quad \text { by } \\
F^{\prime}(U, \xi, \tilde{\varepsilon})=\left[\begin{array}{l}
G\left(A \tilde{\varepsilon} \phi+w, \lambda_{0}+\xi \tilde{\varepsilon}+\mu, \tilde{\varepsilon}^{2}\right) \\
\langle\phi, w\rangle
\end{array}\right]
\end{gathered}
$$

where $A$ is a solution of (4.4) for the given value of $\xi$, and

$$
F^{2}(U, \xi, \tilde{\varepsilon})=\left[\begin{array}{l}
G\left(A \tilde{\varepsilon} \phi+A^{2} \varepsilon^{2} w_{1}+w, \lambda_{0}+\xi \tilde{\varepsilon}^{2}+\mu, \tilde{\varepsilon}^{3}\right) \\
\langle\phi, w\rangle
\end{array}\right]
$$

where $A$ is determined by (4.8) for the given value of $\xi$, and in (4.9) and (4.10) $U=\left(\begin{array}{l}w \\ \mu\end{array}\right) \in H_{1}$.

We consider the equations

$$
F^{\prime}(U, \xi, \tilde{\varepsilon})=0, \quad i=1,2,
$$

corresponding to the two cases 1 and 2, respectively.

For $\xi \in \mathbf{R}$, the linear operator

$$
L_{\iota} \equiv F_{U}^{\prime}(0, \xi, 0)=\left[\begin{array}{ll}
G_{x}^{0} & 0 \\
\langle\phi, \cdot\rangle & 0
\end{array}\right], \quad i=1,2,
$$

satisfies the properties (Lemma 5.6 [3])

(i) $L_{t}$ is a Fredholm operator with zero index,

(ii) $\quad N\left(L_{t}\right)$ is one-dimensional spanned by $\phi_{0}=\left(\begin{array}{l}0 \\ 1\end{array}\right)$,

(iii) $\quad N\left(L_{t}^{*}\right)$ is one-dimensional spanned by $\psi_{0}=\left(\begin{array}{l}\Psi \\ 0\end{array}\right)$,

(iv) the algebraic multiplicity of the zero eigenvalue of $L_{t}$ is two.

It also follows from Lemma 5.6 [3] that for each $C>0$ there exists $\delta(C)>0$ and there are smooth $2 \times 2$ matrices $B_{l}(\tilde{\varepsilon}, \xi), \hat{B}_{l}(\tilde{\varepsilon}, \xi)$ and smooth functions $\phi_{1, j}(\tilde{\varepsilon}, \xi), \psi_{i, j}(\tilde{\varepsilon}, \xi), i, j=1,2$, defined for $|\xi| \leq C$, $|\tilde{\varepsilon}| \leq \delta(C)$ such that the restriction of the linear operators

$$
L_{i}(\tilde{\varepsilon}, \xi) \equiv F_{U}^{l}(0, \xi, \tilde{\varepsilon}), \quad i=1,2,
$$

to the subspace $N_{t}(\tilde{\varepsilon}, \xi)$ spanned by $\phi_{t, 1}(\tilde{\varepsilon}, \xi)$ and $\phi_{t, 2}(\tilde{\varepsilon}, \xi)$ has two eigenvalues which are the same as those of $B_{l}(\tilde{\varepsilon}, \xi)$. Furthermore $H_{1}$ can be written as

$$
H_{1}=N_{\imath}(\tilde{\varepsilon}, \xi) \oplus H_{\imath}(\tilde{\varepsilon}, \xi),
$$

where

$$
H_{i}(\tilde{\varepsilon}, \xi)=\left\{U \in H_{1}:\left\langle\psi_{i, j}(\tilde{\varepsilon}, \xi), U\right\rangle=0, j=1,2\right\},
$$

the restriction of $L_{l}(\tilde{\varepsilon}, \xi)$ to $H_{l}(\tilde{\varepsilon}, \xi)$ has a bounded inverse and the following relations hold 


$$
\begin{gathered}
{\left[\begin{array}{cc}
L_{1}(\tilde{\varepsilon}, \xi) & 0 \\
0 & L_{1}(\tilde{\varepsilon}, \xi)
\end{array}\right]\left[\begin{array}{l}
\phi_{11}(\tilde{\varepsilon}, \xi) \\
\phi_{12}(\tilde{\varepsilon}, \xi)
\end{array}\right]=B_{1}(\tilde{\varepsilon}, \xi)\left[\begin{array}{l}
\phi_{1,1}(\tilde{\varepsilon}, \xi) \\
\phi_{12}(\tilde{\varepsilon}, \xi)
\end{array}\right],} \\
{\left[\begin{array}{cc}
L_{1}^{*}(\tilde{\varepsilon}, \xi) & 0 \\
0 & L_{1}^{*}(\tilde{\varepsilon}, \xi)
\end{array}\right]\left[\begin{array}{l}
\psi_{11}(\tilde{\varepsilon}, \xi) \\
\psi_{1,}(\tilde{\varepsilon}, \xi)
\end{array}\right]=\hat{B}_{1}(\tilde{\varepsilon}, \xi)\left[\begin{array}{l}
\psi_{1,1}(\tilde{\varepsilon}, \xi) \\
\psi_{1,2}(\tilde{\varepsilon}, \xi)
\end{array}\right],} \\
B_{1}(0, \tilde{\xi})=\left[\begin{array}{ll}
0 & 0 \\
1 & 0
\end{array}\right], \quad \hat{B}_{1}(0, \xi)=\left[\begin{array}{ll}
0 & 0 \\
1 & 0
\end{array}\right], \quad \phi_{11}(0, \xi)=\left(\begin{array}{l}
0 \\
1
\end{array}\right) . \\
\phi_{1,2}(0, \xi)=\left(\begin{array}{l}
\phi \\
0
\end{array}\right), \quad \psi_{1,1}(0, \xi)=\left(\begin{array}{l}
\psi \\
0
\end{array}\right) \text { and } \quad \Psi_{12}(0, \xi)=\left(\begin{array}{l}
0 \\
1
\end{array}\right) .
\end{gathered}
$$

for $i=1,2$.

Further properties of the operators defined by (4.14) are given in the following lemmas.

LEMMA 4.1. For $|\xi| \leq C,|\tilde{\varepsilon}| \leq \delta(C)$,

$$
\left\|L_{i}^{-1}(\tilde{\varepsilon}, \xi)\right\|=0\left(\tilde{\varepsilon}^{-1 / 2}\right), \quad i=1,2 .
$$

PROOF. It is enough to show that each eigenvalue $\alpha$ of $B_{l}(\tilde{\varepsilon}, \xi)$ has the form $\alpha=C \tilde{\varepsilon}^{1 / 2}+0(\tilde{\varepsilon})$ for some constant $C \neq 0$. But the solvability condition of the equation resulting by differentiating the first equation of (4.15) with respect to $\tilde{\varepsilon}$ and setting $\tilde{\varepsilon}=0$ gives

$$
\dot{b}_{12}(0, \xi)=A a \neq 0,
$$

where $b_{12}(\tilde{\varepsilon}, \xi)$ is the $(1,2)$-entry of $B_{\imath}(\tilde{\varepsilon}, \xi)$. This shows that $\alpha$ has the desired form.

LEMMA 4.2.

(a) $\left\|L_{1}^{-1}(\tilde{\varepsilon}, \xi) F^{1}(0, \xi, \tilde{\varepsilon})\right\|=0\left(\tilde{\varepsilon}^{5 / 2}\right)$,

(b) $\left\|L_{2}^{-1}(\tilde{\varepsilon}, \xi) F^{2}(0, \xi, \tilde{\varepsilon})\right\|=0\left(\tilde{\varepsilon}^{7 / 2}\right)$.

PROOF. (a) and (b) follow from Lemma 4.1, by expanding $F^{\prime}(0, \xi, \tilde{\varepsilon})$ and observing that $A$ satisfies (4.4), (4.8) for $i=1,2$, respectively.

It follows from Lemmas 4.1 and 4.2 that the functions $h(\tilde{\varepsilon})$ and $q(\tilde{\varepsilon})$ of Theorem 2.4 satisfy

$$
h(\tilde{\varepsilon})=0\left(\tilde{\varepsilon}^{2}\right), \quad q(\tilde{\varepsilon})=0\left(\tilde{\varepsilon}^{2}\right),
$$

for case 1 , and

$$
h(\tilde{\varepsilon})=0\left(\tilde{\varepsilon}^{3}\right), \quad q(\tilde{\varepsilon})=0\left(\tilde{\varepsilon}^{3}\right),
$$

for case 2. This completes the proof of the following theorem.

THEOREM 4.3. For each $C>0$ there exists $\delta(C)>0$ such that for each $\xi$ in $|\xi| \leq C$ and each $\tilde{\varepsilon}$ in $0<|\tilde{\varepsilon}| \leq \delta(C)$ Newton's and chord iterates with initial guess $U^{(0)}=0$ converge to a unique solution of (4.11) for each real solution $A$ of (4.4) or (4.8) (depending upon whether $i=1$ or 2) for the given value of $\xi$.

\section{NUMERICAL EXAMPLE}

In this section we apply the schemes developed in Sections 3 and 4 to the following (finite dimensional) equation.

$$
G\left(\left(\begin{array}{l}
x_{1} \\
x_{2}
\end{array}\right), \lambda, \tau\right)=\left[\begin{array}{l}
x_{2}^{4}+x_{1}^{3}+\frac{3}{2} x_{1} x_{2}^{2}-\lambda x_{1}+\tau \\
x_{1}^{4}+x_{2}^{3}+\frac{1}{2} x_{1}^{2} x_{2}+(1-\lambda) x_{2}
\end{array}\right]=0 .
$$

We notice that for $\tau=0, \lambda=0$ is a simple bifurcation point and properties (1.4) are satisfied with $\phi=\psi=(1,0)^{2}, a=-1, b=1, E_{0}=0$ and $E_{1}=1$, and hence the conditions of case 2 of Section 4 are satisfied. 
Equation (2.1), which determınes the bifurcation curve of (5.1), reduces to

$$
\left.F\left(\begin{array}{l}
y_{1} \\
y_{2} \\
r_{1} \\
r_{2}
\end{array}\right), \varepsilon\right)=\left[\begin{array}{l}
y_{12}^{4}+\varepsilon^{3}+\frac{3}{2} \varepsilon y_{12}^{2}+r_{1} \varepsilon+r_{2} \\
\varepsilon^{4}+y_{12}^{3}+\frac{1}{2} \varepsilon^{2} y_{12}+\left(1-r_{1}\right) y_{12} \\
3 \varepsilon^{2}+\frac{3}{2} y_{12}^{2}+4 y_{12}^{3} y_{22}+3 \varepsilon y_{12} y_{22}-r_{1} \\
4 \varepsilon^{3}+\varepsilon y_{12}+3 y_{12}^{2} y_{22}+\frac{1}{2} \varepsilon^{2} y_{22}+\left(1-r_{1}\right) y_{22}
\end{array}\right]=0,
$$

where $y_{1}=\left(\begin{array}{c}0 \\ y_{12}\end{array}\right)$ and $y_{2}=\left(\begin{array}{c}0 \\ y_{22}\end{array}\right)$. The numerical results obtained by applying the schemes of Section 3 to (5.2) are presented in Table 5.1.

The solution branches of (5.1) through the perturbed bifurcation points are approximated by applyıng the schemes of Section 3 to the equation

$$
K\left(\left(\begin{array}{l}
w_{1} \\
w_{2} \\
\mu
\end{array}\right), \delta\right)=\left[\begin{array}{l}
\left(y_{12}+\delta y_{22}+w_{2}\right)^{4}+\left(\varepsilon+\delta+w_{1}\right)\left[\left(\varepsilon+\delta+w_{1}\right)^{2}+\frac{3}{2}\left(y_{12}+\delta y_{22}+w_{2}\right)^{2}-r_{1}-\mu\right]+r_{2} \\
\left(\varepsilon+\delta+w_{1}\right)^{4}+\left(y_{12}+\delta y_{22}+w_{2}\right)\left[\left(y_{12}+\delta y_{22} w_{2}\right)^{2}+\frac{1}{2}\left(\varepsilon+\delta+w_{1}\right)^{2}+1-r_{1}-\mu\right] \\
w_{1}+y_{22} w_{2}
\end{array}\right]=0 .
$$

The numerical results obtained for the solution branch through the perturbed bifurcation point corresponding to $\varepsilon=0.1$ are presented in Table 5.2.

Finally, we apply the schemes of Section 4 to approximate the solution branch of (5.1) which does not pass through the perturbed bifurcation point.

Equation (4.10) for this example reduces to

$$
F^{2}\left(\left(\begin{array}{c}
0 \\
w_{2} \\
\mu
\end{array}\right), \xi, \tau\right)=\left[\begin{array}{c}
w_{2}^{4}+x_{1}^{3}+\frac{3}{2} x_{1} w_{2}^{2}-\left(\xi \tau^{2 / 3}+\mu\right) x_{1}+\tau \\
x_{1}^{4}+w_{2}^{3}+\frac{1}{2} x_{1}^{2} w_{2}+\left(1-\xi \tau^{2 / 3}-\mu\right) w_{2}
\end{array}\right]=0,
$$

where $x_{1}=A \tau^{1 / 3}\left(1+A \tau^{1 / 3}\right)$ and $A$ is the negative root of $A^{3}-\xi A+1=0$. The numerical results obtained for $\tau=.002$ are presented in Table 5.3.

The numerical results of Tables 5.2 and 5.3 determine approximations to all the solution branches near the perturbed bifurcation point when $\tau=.002$, which corresponds to $\varepsilon=0.1$ in Table 5.1 .

In Tables 5.1-5.3, $N$ and $C$ denote the number of iterations needed for the convergence of Newton's and chord methods respectively.

Table 5.1

\begin{tabular}{|c|cccc|c|r|}
\hline$\varepsilon$ & $\left(y_{12}\right.$, & $y_{22}$, & $r_{1}$, & $\left.r_{2}\right)$ & $N$ & $C$ \\
\hline-0.20 & $(-1.8(-03)$, & $3.5(-02)$, & $1.2(-01)$, & $-1.6(-02))$ & 5 & 17 \\
-0.15 & $(-5.4(-04)$, & $1.4(-02)$, & $6.8(-02)$, & $-6.8(-03))$ & 4 & 13 \\
-0.10 & $(-1.0(-04)$, & $4.1(-03)$, & $3.0(-02)$, & $-2.0(-03))$ & 4 & 10 \\
-0.05 & $(-6.3(-06)$, & $5.0(-04)$, & $7.5(-03)$, & $-2.5(-04))$ & 4 & 7 \\
0.00 & $(00$, & 0, & $0,$, & $0)$ & 0 & 0 \\
0.05 & $(-6.3(-06)$, & $-5.0(-04)$, & $7.5(-03)$, & $2.5(-04))$ & 4 & 7 \\
0.10 & $(-1.0(-04)$, & $-4.1(-03)$, & $3.0(-02)$, & $2.0(-03))$ & 4 & 10 \\
0.15 & $(-5.4(-04)$, & $-1.4(-02)$, & $6.8(-02)$, & $6.8(-03))$ & 4 & 13 \\
0.20 & $(-1.8(-03)$, & $-3.5(-02)$, & $1.2(-01)$, & $1.6(-02))$ & 5 & 17 \\
\hline
\end{tabular}


Table 5.2

\begin{tabular}{|c|ccc|c|c|}
\hline$\delta$ & $\left(r w_{1}\right.$, & $w_{2}$, & $\mu)$ & $N$ & $C$ \\
\hline-0.08 & $(-9.2(-07)$, & $-2.2(-04)$, & $7.0(-02))$ & 4 & 12 \\
-0.06 & $(-6.0(-07)$, & $-1.5(-04)$, & $2.2(-02))$ & 4 & 9 \\
-0.04 & $(-3.1(-07)$, & $-7.5(-05)$, & $6.9)-03))$ & 4 & 7 \\
-0.02 & $(-8.8(-08)$, & $-2.1(-05)$, & $1.4(-03))$ & 4 & 5 \\
0.00 & $(0$ & 0 & $0)$ & 0 & 0 \\
0.02 & $(-1.1(-07)$, & $-2.8(-05)$, & $1.1(-03))$ & 4 & 5 \\
0.04 & $(-5.2(-07)$, & $-1.3(-04)$, & $3.9(-03))$ & 4 & 7 \\
0.06 & $(-1.3(-06)$, & $-3.2(-04)$, & $8.1(-03))$ & 4 & 8 \\
0.08 & $(-2.7(-06)$, & $-6.5(-04)$, & $1.4(-02))$ & 4 & 8 \\
\hline
\end{tabular}

Table 5.3

\begin{tabular}{|c|c|c|c|c|c|c|}
\hline$\xi$ & ( & $w_{1}$, & $w_{2}$, & $\mu$ & $N$ & C \\
\hline-5.0 & $(0$, & & $-3.3(-07)$ & $-2.1(-03))$ & 2 & 2 \\
\hline-4.0 & $(0$, & & $-7.7(-07)$ & $-2.1(-03))$ & 2 & 2 \\
\hline-3.0 & $(0$, & & $-2.2(-06)$ & $-2.2(-03))$ & 2 & 2 \\
\hline-2.0 & $(0$, & & $-8.1(-06)$, & $-2.5(-03))$ & 2 & 2 \\
\hline-1.0 & $(0$, & & $-3.7(-05)$ & $-3.4(-03))$ & 3 & 3 \\
\hline 0.0 & $(0$, & & $-1.5(-04)$ & $-6.0(-03))$ & 3 & 3 \\
\hline 1.0 & $(0$, & & $-3.7(-04)$, & $-1.1(-02))$ & 3 & 3 \\
\hline 2.0 & $(0$, & & $-6.9(-04)$, & $-1.8(-02))$ & 3 & 4 \\
\hline 3.0 & $(0$, & & $-1.1(-03)$, & $-2.6(-02))$ & 3 & 4 \\
\hline 4.0 & $(0$, & & $-1.5(-03)$ & $-3.6(-02))$ & 3 & 4 \\
\hline 5.0 & $(0$, & & $-1.9(-03)$ & $-4.6(-02))$ & 3 & 5 \\
\hline 6.0 & $(0$, & & $-2.3(-03)$, & $-5.7(-02))$ & 3 & 5 \\
\hline 7.0 & $(0$, & & $-2.6(-03)$ & $-6.9(-02))$ & 3 & 5 \\
\hline 8.0 & $(0$, & & $-2.9(-03)$ & $-8.2(-02))$ & 4 & 6 \\
\hline 9.0 & $(0$, & & $-3.2(-03)$ & $-9.5(-02))$ & 4 & 6 \\
\hline
\end{tabular}

ACKNOWLEDGMENT. The authors are grateful to Mrs. Sue Johnson for typing the manuscript of this paper.

\section{REFERENCES}

[1] BOHL, E., "Chord Techniques and Newton's Method for Discrete Bifurcation Problems," Numer. Math., 34 (1980), 111-124.

[2] CRANDALL, M. G. and RABINOWITZ, P. H., "Bifurcation from Simple Eigenvalues," $J$. Funct. Anal., 8 (1971), 321-340.

[3] DECKER, D. W. and KELLER, H. B., "Path Following Near Bifurcation," Comm. on Pure and Applied Math., 34 (1981), 149-175.

[4] GOLUBITSKY, M. and SCHAEFFER, D., "A Theory for Imperfect Bifurcation via Singularity Theory," Comm. Math., 32 (1979), 21-98.

[5] KATO, T., Perturbation Theory of Linear Operators, Springer, 1976.

[6] KELLER, H. B., "Numerical Solution of Bifurcation and Nonlinear Eigenvalue Problems," Application of Bifurcation Theory, Rabinowitz, P. H., ed., Academic Press, New York (1977), 359-384.

[7] KELLER, H. B. and KEENER, J., "Perturbed Bifurcation Theory," Arch. Mech. Anal., 50 (1973), 159-175. 
[8] MATKOWSKY, B. J. and REISS, E. L., "Singular Perturbation of Bifurcation," SIAM J. Appl. Math., 33, 2 (1977), 230-255.

[9] MOORE, G., "The Numerical Treatment of Non-Trivial Bifurcation Points," Numer. Funct. Anal. and Optimiz., 2(6) (1980), 441-472.

[10] MOORE, G., "The Application of Newton's Method to Simply Bifurcation and Turning Points Problems," Ph.D. Thests, University of Bath, 1979.

[11] REISS, E. L., "Imperfect Bifurcation," Application of Bifurcation Theory. Rabınowitı, P. H., ed., Academic Press, New York, 1977, 37-71. 


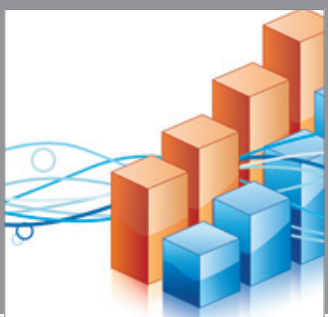

Advances in

Operations Research

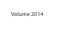

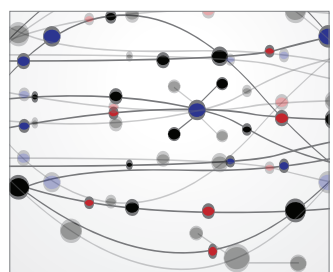

\section{The Scientific} World Journal
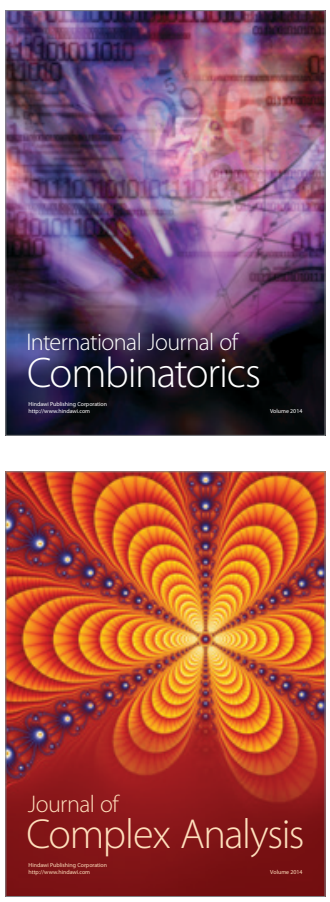

International Journal of

Mathematics and

Mathematical

Sciences
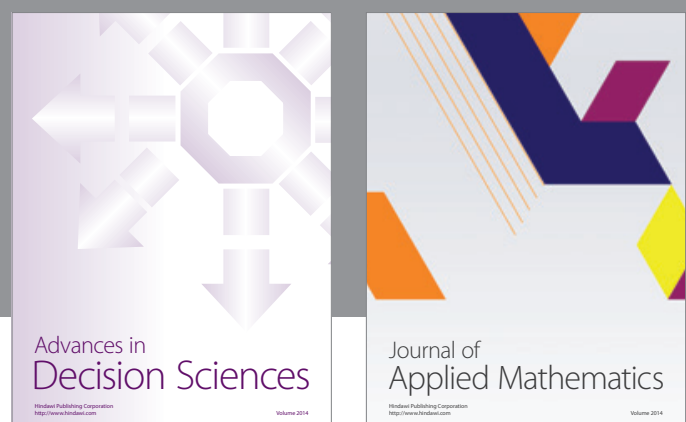

Journal of

Applied Mathematics
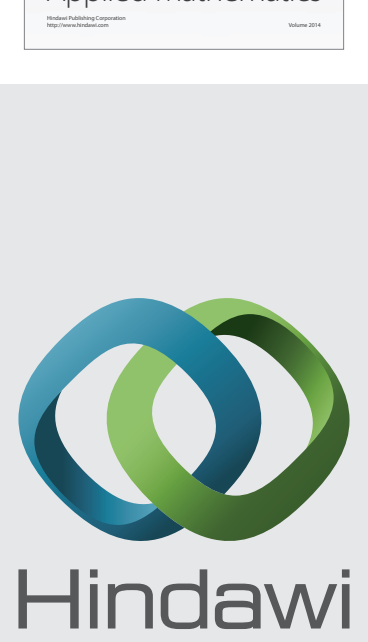

Submit your manuscripts at http://www.hindawi.com
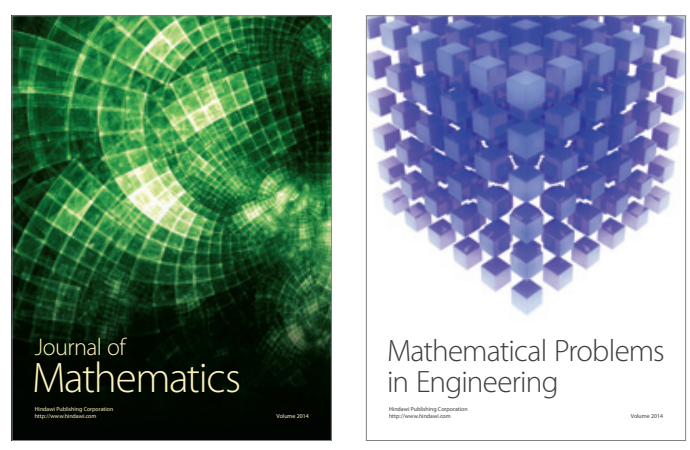

Mathematical Problems in Engineering
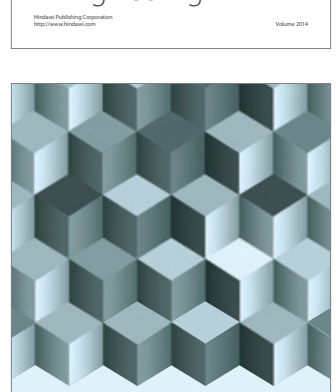

Journal of

Function Spaces
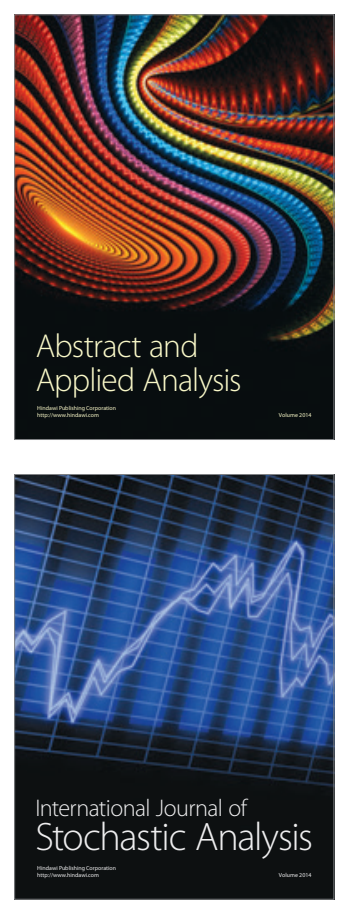

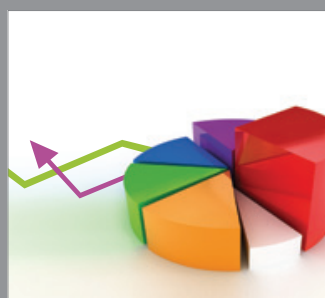

ournal of

Probability and Statistics

Promensencen
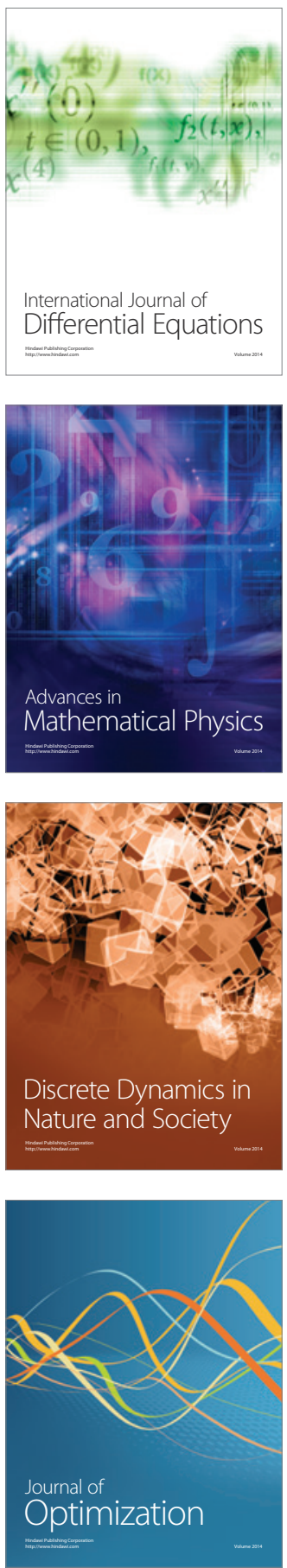